\section{- OPEN ACCESS}

- Additional material is published online only. To view please visit the journal online (http://dx.doi.org/10.1136/ heartjnl-2013-304739).

For numbered affiliations see end of article.

\section{Correspondence to} Professor Yukihito Higashi, Department of Cardiovascular Regeneration and Medicine, Research Institute for Radiation Biology and Medicine, Hiroshima University, 1-2-3 Kasumi, Minami-ku, Hiroshima 734-8551, Japan; yhigashi@hiroshima-u.ac.jp

Received 30 July 2013 Revised 11 September 2013 Accepted 12 September 2013 Published Online First 24 October 2013

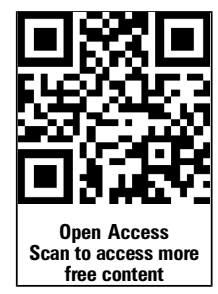

To cite: Maruhashi $\mathrm{T}$, Soga J, Fujimura N, et al. Heart 2013;99:1837-1842.

\title{
Relationship between flow-mediated vasodilation and cardiovascular risk factors in a large community-based study
}

\author{
Tatsuya Maruhashi, ${ }^{1}$ Junko Soga, ${ }^{1}$ Noritaka Fujimura, ${ }^{1}$ Naomi Idei, ${ }^{1}$ \\ Shinsuke Mikami, ${ }^{1}$ Yumiko Iwamoto, ${ }^{1}$ Masato Kajikawa, ${ }^{1}$ Takeshi Matsumoto, ${ }^{1}$ \\ Takayuki Hidaka, ${ }^{1}$ Yasuki Kihara, ${ }^{1}$ Kazuaki Chayama, ${ }^{2}$ Kensuke Noma, ${ }^{3,4}$ \\ Ayumu Nakashima, ${ }^{4}$ Chikara Goto, ${ }^{5}$ Hirofumi Tomiyama, ${ }^{6}$ Bonpei Takase, ${ }^{7}$ \\ Akira Yamashina, ${ }^{6}$ Yukihito Higashi ${ }^{3,4}$
}

\section{ABSTRACT \\ Objective To determine the relationships between} flow-mediated vasodilation (FMD) and cardiovascular risk factors, and to evaluate confounding factors for measurement of FMD in a large general population in Japan.

Methods This was a cross-sectional study. A total of 5314 Japanese adults recruited from people who underwent health screening from 1 April 2010 to 31 August 2012 at 3 general hospitals in Japan. Patients' risk factors (age, Body Mass Index, blood pressure, cholesterol parameters, glucose level and $\mathrm{HbA1c}$ level) and prevalence of cardiovascular disease (coronary heart disease and cerebrovascular disease) were investigated. Results Univariate regression analysis revealed that FMD correlated with age $(r=-0.27, p<0.001)$, Body Mass Index $(r=-0.14, p<0.001)$, systolic blood pressure $(r=-0.18, p<0.001)$, diastolic blood pressure $(r=-0.13$, $p<0.001)$, total cholesterol $(r=-0.07, p<0.001)$, triglycerides $(r=-0.10, p<0.001)$, high-density lipoprotein cholesterol $(r=0.06, p<0.001)$, low-density lipoprotein cholesterol $(r=-0.04, p=0.01)$, glucose level $(r=-0.14, p<0.001), \operatorname{HbA1c}(r=-0.14, p<0.001)$, and baseline brachial artery diameter $(r=-0.43, p<0.001)$ as well as Framingham Risk score $(r=-0.29, p<0.001)$. Multivariate analysis revealed that age ( $\mathrm{t}$ value $=-9.17$, $p<0.001$ ), sex (t value $=9.29, p<0.001$ ), Body Mass Index (t value $=4.27, p<0.001$ ), systolic blood pressure ( $\mathrm{t}$ value $=-2.86, \mathrm{p}=0.004$ ), diabetes mellitus ( $\mathrm{t}$ value $=$ $-4.19, p<0.001$ ), smoking (t value $=-2.56, p=0.01$ ), and baseline brachial artery diameter ( $\mathrm{t}$ value $=-29.4$, $p<0.001$ ) were independent predictors of FMD.

Conclusions FMD may be a marker of the grade of atherosclerosis and may be used as a surrogate marker of cardiovascular outcomes. Age, sex, Body Mass Index, systolic blood pressure, diabetes mellitus, smoking and, particularly, baseline brachial artery diameter are potential confounding factors in the measurement of FMD.

\section{INTRODUCTION}

Endothelial dysfunction is established in the initial step of atherosclerosis and plays an important role in the development of this condition. ${ }^{1}$ Additionally, it is well known that endothelial function is an independent predictor of cardiovascular events. ${ }^{2}$
Therefore, it is clinically important to estimate the condition of endothelial function, which provides additive information for risk stratification of patients. Recently, measurement of flow-mediated vasodilation (FMD) in the brachial artery using high-resolution ultrasound has been widely used as a method for assessing endothelial function. ${ }^{3-7}$ Measurement of FMD reflects nitric oxide (NO) production, and growing evidence has shown that endothelial function assessed by FMD can serve as an independent predictor of cardiovascular events. ${ }^{8-10}$ However, methodology for measuring FMD has not been fully established. ${ }^{11}{ }^{12}$ In the traditional approach, FMD is assessed at arbitrary time points after cuff release, ${ }^{3} 51314$ whereas, current technological advancement enables evaluation of continuous changes in brachial artery diameter automatically using ultrasonography with an edge detection and wall tracking system, providing true peak diameter during reactive hyperaemia. Indeed, a recent study has demonstrated that the time to peak diameter was different in young and older subjects, and that FMD calculated at a single time point underestimates the true peak FMD. ${ }^{15}$ In addition to methodological issues, the absence of diagnostic criteria makes it difficult to interpret the results of FMD in clinical practice.

Although several investigators have examined the relationships between FMD and cardiovascular risk factors as well as potential factors that might confound interpretation of FMD, previous studies were limited to small numbers of or highly selected subjects, and used the traditional approach in which FMD was calculated at arbitrary time points or within a fixed time window. ${ }^{4}{ }^{13}{ }^{16}$ We therefore investigated the relationships between FMD and cardiovascular risk factors in a large general population, and we evaluated correlated factors for measurement of FMD using automated computerised ultrasonography with an edge-tracking system that enables continuous assessment of brachial artery diameter.

\section{METHODS}

\section{Subjects}

A total of 5314 Japanese adults aged $17-86$ years who underwent health-screening examinations with 
agreement for measurement of vascular function were enrolled in the Flow-mediated Dilation Japan Registry between 1 April 2010 and 31 August 2012 at three general hospitals in Japan. All employees have an obligation to undergo health screening every year under the regulations of the society-managed health insurance union in Japan. In accordance with the regulations, we performed health-screening examinations. Subjects with severe valvular heart disease, arrhythmia that required treatment, severe chronic heart failure (New York Heart Association level of more than III), or malignancy and patients with receiving treatment with steroids, non-steroidal anti-inflammatory drugs or immunosuppressive drugs were excluded from the study. Hypertension was defined as systolic blood pressure of more than $140 \mathrm{~mm} \mathrm{Hg}$ or diastolic blood pressure of more than $90 \mathrm{~mm} \mathrm{Hg}$, in a sitting position, on at least three different occasions. Patients with secondary forms of hypertension were excluded on the basis of complete history; physical examination; radiological and ultrasound examinations; urinalysis; plasma rennin activity; plasma aldosterone and norepinephrine concentrations; serum creatinine, potassium, calcium and free thyroxine concentrations; and 24-h urinary excretion of 17-hydroxycorticosteroids, 17-ketogenic steroids and vanillymandelic acid. Diabetes was defined according to the American Diabetes Association. ${ }^{17}$ Dyslipidemia was defined according to the third report of the National Cholesterol Education Program. ${ }^{18}$ We defined smokers as those who were currently smoking. Coronary heart disease included angina pectoris, myocardial infarction and unstable angina. Unstable angina was designated when a history of prolonged ischaemic chest pain (>15 min in duration) was accompanied by transient ischaemic ST segment and T-wave abnormality in the electrocardiographic tracing but not accompanied by development of Q-wave abnormality or by serum enzyme changes characteristic of myocardial necrosis. Cerebrovascular disease included ischaemic stroke, haemorrhagic stroke, and transient ischaemic attack. Healthy subjects had no history of cardiovascular and cerebrovascular diseases, liver diseases, renal diseases, autoimmune diseases, or malignant diseases and had no coronary risk factors, including hypertension, dyslipidemia, diabetes mellitus and smoking. Framingham Risk score was calculated by points of risk factors: age, total cholesterol level, high-density lipoprotein (HDL) cholesterol level, systolic blood pressure and smoking status. ${ }^{19}$ The ethical committees of our institutions approved the study protocol. Written informed consent for participation in the study was obtained from all subjects.

Subjects fasted the previous night for at least $12 \mathrm{~h}$. After remaining in the supine position for $30 \mathrm{~min}$, fasting serum concentrations of total cholesterol, HDL cholesterol, low-density lipoprotein (LDL) cholesterol, triglycerides, creatinine, glucose and $\mathrm{HbA} 1 \mathrm{c}$ were measured.

\section{Measurement of FMD}

All studies were performed in the morning, after overnight fasting, in a quiet, dark and air-conditioned room (constant temperature of $22-25^{\circ} \mathrm{C}$ ). The subjects remained supine throughout the study. The vascular response to reactive hyperaemia in the brachial artery was used for assessment of endotheliumdependent FMD. For all details on the measurements, see the online-only supplementary data.

\section{Statistical analysis}

Results are presented as mean \pm SD. All reported probability values were two-sided, and a probability value of $<0.05$ was considered statistically significant. Categorical variables were compared by means of $\chi^{2}$ test. Continuous variables were compared by using analysis of variance (ANOVA) for multiple groups. Relations between variables were determined by Spearman correlation coefficients analysis. Multivariate regression analyses were performed to identify factors associated with FMD in risk factors and laboratory data. The data were processed using the software package Stata V.9 (Stata, College Station, Texas, USA).

\section{RESULTS}

\section{Baseline clinical characteristics}

The baseline clinical characteristics of the subjects are summarised in table 1 . The age range was $17-86$ years. Of the 5314 subjects, 4131 (77.7\%) were men and 1183 (22.3\%) were women. Seven hundred and thirty-eight (13.9\%) had hypertension, 2264 (42.6\%) had dyslipidemia, 336 (6.3\%) had diabetes mellitus and $1495(28.4 \%)$ were smokers. The mean value of FMD was 6.16 $3.26 \%$ (median, 5.9\%; IQR, 4.0-8.0\%; range, $-4.7-21.9 \%$ ) (see online supplementary figure S1). FMD was significantly lower in men than in women $(5.92 \pm 3.05$ vs 6.99 $\pm 3.77 \%, \mathrm{p}<0.001)$. Baseline brachial artery diameter was significantly larger in men than in women $(4.19 \pm 0.50$ vs 3.38 $\pm 0.48 \mathrm{~mm}, \mathrm{p}<0.001)$.

\section{Relationships between FMD and cardiovascular risk factors}

There was a significant negative correlation between FMD and Framingham Risk score $(\mathrm{r}=-0.29, \mathrm{p}<0.001)$ (see online supplementary figure S2A). Univariate regression analysis revealed that FMD significantly correlated with age $(r=-0.27$, $\mathrm{p}<0.001)$, Body Mass Index $(\mathrm{r}=-0.14, \mathrm{p}<0.001)$, systolic blood pressure $(\mathrm{r}=-0.18, \mathrm{p}<0.001)$, diastolic blood pressure $(\mathrm{r}=-0.13, \mathrm{p}<0.001)$, total cholesterol $(\mathrm{r}=-0.07, \mathrm{p}<0.001)$, triglycerides $(\mathrm{r}=-0.10, \mathrm{p}<0.001)$, HDL cholesterol $(\mathrm{r}=0.06$, $\mathrm{p}<0.001)$, LDL cholesterol $(\mathrm{r}=-0.04, \mathrm{p}=0.01)$, glucose level $(\mathrm{r}=-0.14, \mathrm{p}<0.001), \mathrm{HbA1} \mathrm{c}(\mathrm{r}=-0.14, \mathrm{p}<0.001)$, and baseline brachial artery diameter $(\mathrm{r}=-0.43, \mathrm{p}<0.001)$, as well as

Table 1 Clinical characteristics of the subjects

\begin{tabular}{ll}
\hline Variables & $\mathrm{n}=5314$ \\
\hline Age (years) & $46 \pm 13$ \\
Male, $\mathrm{n}(\%)$ & $4135(77.7)$ \\
Body Mass Index, kg/m² & $23.0 \pm 3.3$ \\
Systolic blood pressure, mm Hg & $124.6 \pm 17.3$ \\
Diastolic blood pressure, mm Hg & $76.1 \pm 13.0$ \\
Heart rate, bpm & $64.0 \pm 10.5$ \\
Total cholesterol, mmol/L & $5.23 \pm 0.88$ \\
Triglycerides, mmol/L & $1.43 \pm 1.05$ \\
HDL cholesterol, mmol/L & $1.57 \pm 0.42$ \\
LDL cholesterol, mmol/L & $3.19 \pm 0.81$ \\
Glucose, mmol/L & $5.53 \pm 1.18$ \\
HbA1c, \% & $5.1 \pm 0.9$ \\
Hypertension, $\mathrm{n}$ (\%) & $738(13.9)$ \\
Dyslipidemia, $\mathrm{n}(\%)$ & $2264(42.6)$ \\
Diabetes mellitus, $\mathrm{n}$ (\%) & $336(6.3)$ \\
Smoking, $\mathrm{n}(\%)$ & $1495(28.4)$ \\
Coronary heart disease, $\mathrm{n}(\%)$ & $117(2.3)$ \\
Cerebrovascular disease, $\mathrm{n}(\%)$ & $46(1.0)$ \\
Flow-mediated vasodilation, \% & $6.16 \pm 3.26$ \\
Baseline brachial artery diameter, mm & $4.01 \pm 0.60$ \\
\hline HDL, high-density lipoprotein cholesterol; LDL, low-density lipoprotein cholesterol.
\end{tabular}


Table 2 Clinical characteristics of four quartiles of FMD

\begin{tabular}{|c|c|c|c|c|c|}
\hline \multirow[b]{2}{*}{ Variables } & \multicolumn{4}{|c|}{ FMD quartiles } & \multirow[b]{2}{*}{ p Value } \\
\hline & $\begin{array}{l}\text { Quartile } 1 \\
\leq 3.9 \\
(n=1329)\end{array}$ & $\begin{array}{l}\text { Quartile } 2 \\
4.0-5.8 \\
(n=1329)\end{array}$ & $\begin{array}{l}\text { Quartile } 3 \\
5.9-7.9 \\
(n=1328)\end{array}$ & $\begin{array}{l}\text { Quartile } 4 \\
\geq 8.0 \\
(n=1328)\end{array}$ & \\
\hline Age (years) & $50.6 \pm 13.2$ & $47.7 \pm 12.8$ & $44.8 \pm 12.9$ & $42.0 \pm 12.8$ & $<0.001$ \\
\hline Body Mass Index, $\mathrm{kg} / \mathrm{m}^{2}$ & $23.5 \pm 3.3$ & $23.2 \pm 3.2$ & $23.0 \pm 3.3$ & $22.3 \pm 3.2$ & $<0.001$ \\
\hline Systolic blood pressure, $\mathrm{mm} \mathrm{Hg}$ & $128.5 \pm 18.5$ & $125.9 \pm 17.1$ & $123.6 \pm 16.3$ & $120.5 \pm 15.9$ & $<0.001$ \\
\hline Diastolic blood pressure, $\mathrm{mm} \mathrm{Hg}$ & $77.8 \pm 13.1$ & $77.2 \pm 13.0$ & $75.6 \pm 12.5$ & $73.9 \pm 12.8$ & $<0.001$ \\
\hline Heart rate, bpm & $64.1 \pm 11.0$ & $63.9 \pm 10.9$ & $63.4 \pm 10.0$ & $64.6 \pm 9.9$ & 0.03 \\
\hline Total cholesterol, mmol/L & $5.30 \pm 0.89$ & $5.29 \pm 0.87$ & $5.25 \pm 0.89$ & $5.18 \pm 0.86$ & 0.01 \\
\hline Triglycerides, mmol/L & $1.57 \pm 1.20$ & $1.48 \pm 1.05$ & $1.38 \pm 0.90$ & $1.29 \pm 1.01$ & $<0.001$ \\
\hline HDL cholesterol, mmol/L & $1.55 \pm 0.43$ & $1.55 \pm 0.41$ & $1.57 \pm 0.40$ & $1.61 \pm 0.42$ & 0.01 \\
\hline LDL cholesterol, mmol/L & $3.20 \pm 0.89$ & $3.21 \pm 0.77$ & $3.20 \pm 0.78$ & $3.14 \pm 0.78$ & 0.14 \\
\hline Glucose, $\mathrm{mmol} / \mathrm{L}$ & $5.73 \pm 1.64$ & $5.59 \pm 1.17$ & $5.46 \pm 0.85$ & $5.35 \pm 0.83$ & $<0.001$ \\
\hline $\mathrm{HbA1c}, \%$ & $5.24 \pm 0.75$ & $5.17 \pm 0.57$ & $5.10 \pm 0.52$ & $5.04 \pm 0.46$ & $<0.001$ \\
\hline Framingham Risk score & $4.36 \pm 4.34$ & $3.37 \pm 4.83$ & $2.08 \pm 5.42$ & $0.57 \pm 6.39$ & $<0.001$ \\
\hline Baseline brachial artery diameter, mm & $4.32 \pm 0.61$ & $4.11 \pm 0.53$ & $3.92 \pm 0.53$ & $3.66 \pm 0.52$ & $<0.001$ \\
\hline FMD, \% & $2.33 \pm 1.40$ & $4.97 \pm 0.54$ & $6.94 \pm 0.59$ & $10.41 \pm 2.23$ & $<0.001$ \\
\hline Hypertension, n (\%) & $331(25.1)$ & $196(14.8)$ & $133(10.0)$ & $78(5.9)$ & $<0.001$ \\
\hline Dyslipidemia, n (\%) & $655(49.6)$ & $598(45.2)$ & $539(40.6)$ & $472(35.8)$ & $<0.001$ \\
\hline Diabetes mellitus, n (\%) & $134(10.2)$ & $91(6.9)$ & $67(5.1)$ & $44(3.3)$ & $<0.001$ \\
\hline Smoking, n (\%) & $461(35.1)$ & $407(30.9)$ & $346(26.1)$ & $281(21.4)$ & $<0.001$ \\
\hline Coronary heart disease, n (\%) & $47(3.6)$ & $30(2.3)$ & $24(1.8)$ & $16(1.3)$ & $<0.001$ \\
\hline Cerebrovascular disease, n (\%) & $20(1.9)$ & $12(1.1)$ & $8(0.7)$ & $6(0.5)$ & 0.01 \\
\hline
\end{tabular}

Framingham Risk score (see online supplementary table S1). Subjects were classified into four groups based on Framingham Risk scores: first quartile $(\leq 0)$, second quartile (1-3), third quartile (4-6), and fourth quartile $(\geq 7)$. FMD decreased in relation to increase in the Framingham Risk score $(7.32 \pm 3.46 \%, 6.28$ $\pm 3.06 \%, 5.75 \pm 2.94 \%$, and $4.93 \pm 2.88 \%, \mathrm{p}<0.001$, respectively, see online supplementary figure $\mathrm{S} 2 \mathrm{~B}$ ).

Online supplementary figure S3 shows FMD in subjects with no cardiovascular risk factors (no-risk group), subjects with at least one coronary risk factor, including hypertension, dyslipidemia, diabetes mellitus and smoking, but without established cardiovascular disease (at-risk group), and subjects with cardiovascular disease (CVD group). FMD in the CVD group was significantly impaired compared to that in the no-risk group or at-risk group (no-risk group, $6.70 \pm 3.25 \%$; at-risk group, $5.45 \pm 3.03 \%$; CVD group, $4.88 \pm 3.00 \%$; $<<0.001$ for CVD group vs no-risk group; $\mathrm{p}=0.03$ for CVD group vs at-risk group), and FMD in the at-risk group was significantly impaired compared with that in the no-risk group $(p<0.001)$.

We next categorised subjects into four groups based on FMD (table 2). There were significant decreases in the prevalence of hypertension, dyslipidemia, diabetes mellitus, smoking, coronary heart disease and cerebrovascular disease with increase in FMD (see online supplementary figure S4A and S4B). Multivariate analysis revealed that age, sex, Body Mass Index, systolic blood pressure, diastolic blood pressure, dyslipidemia, diabetes mellitus, smoking and baseline brachial artery diameter were independent predictors of FMD (table 3).

\section{Relationships of FMD with age and gender}

Subjects were divided into six groups based on age decades from the 20s through the 70s. FMD was significantly higher in women than in men from the 20 s until the 40 s $(8.77 \pm 3.55$ vs $6.63 \pm 3.28 \%$ in the $20 \mathrm{~s} ; 8.47 \pm 3.55 \%$ vs $6.60 \pm 2.91 \%$ in the $30 \mathrm{~s} ; 7.51 \pm 3.72$ vs $6.24 \pm 3.04 \%$ in $40 \mathrm{~s} ; \mathrm{p}<0.001$, respectively), after which it declined. There was no significant gender difference in FMD from the 50 s $(5.85 \pm 3.43$ vs $5.56 \pm 2.90 \%$ in the $50 \mathrm{~s} ; 4.45 \pm 2.80$ vs $5.05 \pm 2.93 \%$ in the $60 \mathrm{~s} ; 4.53 \pm 2.65$ vs 3.83 $\pm 2.28 \%$ in over $70 \mathrm{~s}$ ) in all subjects (see online supplementary figure $\mathrm{S} 5 \mathrm{~A}$ ). In the no-risk group, FMD was significantly higher in women than in men from the $20 \mathrm{~s}$ until the $40 \mathrm{~s}(8.75 \pm 3.54$ vs $6.64 \pm 3.07 \%$ in the $20 \mathrm{~s} ; 8.60 \pm 3.43$ vs $6.61 \pm 2.87 \%$ in the $30 \mathrm{~s} ; 7.78 \pm 3.80$ vs $6.60 \pm 3.08 \%$ in $40 \mathrm{~s} ; \mathrm{p}<0.001$, respectively, online supplementary figure S5B), after which it declined. There was no significant gender difference in FMD from the 50s $(6.10$ \pm 3.26 vs $5.94 \pm 2.88 \%$ in the $50 \mathrm{~s} ; 5.32 \pm 2.61$ vs $5.79 \pm 3.01 \%$ in the $60 \mathrm{~s} ; 5.55 \pm 2.61$ vs $4.00 \pm 2.63 \%$ in over $70 \mathrm{~s})$. On the other hand, in the at-risk and CVD groups, FMD was

Table 3 Multivariate analysis of the relation between flow-mediated vasodilation and variables

\begin{tabular}{lcrc}
\hline Variables & $\boldsymbol{\beta}$ & t Value & $\boldsymbol{p}$ Value \\
\hline Age (years) & -0.14 & -9.68 & $<0.001$ \\
Male & 0.14 & 9.17 & $<0.001$ \\
Body Mass Index, kg/m ${ }^{2}$ & 0.052 & 3.66 & $<0.001$ \\
Systolic blood pressure, mm Hg & -0.12 & -5.85 & $<0.001$ \\
Diastolic blood pressure, mm Hg & 0.11 & 5.32 & $<0.001$ \\
Dyslipidemia & -0.013 & -0.99 & 0.32 \\
Diabetes mellitus & -0.046 & -3.61 & $<0.001$ \\
Smoking & -0.034 & -2.62 & 0.009 \\
Baseline brachial artery diameter, mm & -0.47 & -29.4 & $<0.001$ \\
\hline
\end{tabular}


significantly higher in women than in men in the 20s $(9.64$ \pm 5.54 vs $6.05 \pm 3.65 \%, p<0.001)$ but was not significantly different in women and men from the $30 \mathrm{~s}$ through the $70 \mathrm{~s}(7.54$ \pm 4.53 vs $6.48 \pm 2.95 \%$ in the $30 \mathrm{~s} ; 6.25 \pm 3.13$ vs $5.86 \pm 2.95 \%$ in the $40 \mathrm{~s} ; 5.34 \pm 3.72$ vs $5.27 \pm 2.85 \%$ in the $50 \mathrm{~s} ; 3.84 \pm 2.83$ vs $4.09 \pm 2.81 \%$ in the $60 \mathrm{~s} ; 4.10 \pm 2.59$ vs $3.86 \pm 2.23 \%$ in over $70 \mathrm{~s}$ ) (see online supplementary figure S5C). Baseline clinical characteristics of subjects for each decade of age, baseline clinical characteristics of men and women, and results of univariate analysis for the relationships between FMD and variables in men and women are summarised in online supplementary tables II-IV.

\section{Relationships between FMD and blood pressure}

Subjects were classified into four groups on the basis of the criteria of Joint National Committee (JNC) $\mathrm{VII}^{20}$ : normal, prehypertension, stage 1 hypertension and stage 2 hypertension. The clinical characteristics of blood pressure categories are summarised in online supplementary table V. FMD was significantly higher in the normal blood pressure group than in the prehypertension group and decreased significantly in relation to increase in the stage of blood pressure category (see online supplementary figure S6).

\section{Relationship between FMD and baseline brachial artery diameter}

We classified subjects into four groups based on the baseline brachial artery diameter (quartile 1, $\leq 3.60 \mathrm{~mm}$; quartile 2, $3.61-\leq 4.03 \mathrm{~mm}$; quartile $3,4.04-\leq 4.41 \mathrm{~mm}$; and quartile 4 , $\geq 4.42 \mathrm{~mm}$ ). The clinical characteristics of subjects in the brachial artery diameter quartiles are summarised in online supplementary table VI. Figure 1 shows the relationship between FMD and baseline brachial artery diameter in each decade of age. FMD decreased significantly in relation to increase in baseline brachial artery diameter and correlated negatively with baseline brachial artery diameter in every decade from the 20s through the over 70s (see online supplementary table VII). On a gender basis, there were significant correlations between FMD and baseline brachial artery diameter in men and women (see online supplementary table VIII).

\section{DISCUSSION}

In the present study, we demonstrated that FMD decreased in relation to cumulative cardiovascular risk factors and significantly correlated with cardiovascular risk factors. Additionally, age, sex, blood pressure and, particularly, baseline brachial artery diameter were strong independent predictors of FMD. Although correlations between FMD and coronary risk factors were investigated in previous studies, ${ }^{3-5}{ }^{13}$ FMD was measured at an arbitrary time point, typically 60 s after cuff release, or within a fixed time window during reactive hyperaemia in those subjects. However, these methods have been demonstrated to have the possibility of failing to identify the true peak diameter, leading to underestimation of FMD values and drawing a different conclusion. ${ }^{15}$ A recently developed ultrasound system enables continuous assessment of brachial artery diameter change. In the present study, we adopted a continuous diameter assessment methodology using automated computerised ultrasonography with an edge-tracking system. In this approach, the transducer was held at the same position, and edges of the brachial artery were automatically detected and continuously tracked at the same point throughout the study. Therefore, compared with the traditional approach, this method may provide true values of peak FMD and enable more accurate and simpler measurement of FMD.

It is well known that cardiovascular risk factors impair endothelial function. ${ }^{1}$ In the present study, we confirmed that endothelial function assessed by FMD significantly correlated with cardiovascular risk factors, including age, Body Mass Index, systolic and diastolic blood pressure, total cholesterol, triglycerides, HDL cholesterol, LDL cholesterol, glucose and HbA1c, and that prevalence of hypertension, dyslipidemia, diabetes mellitus and smoking, as well as the incidence of CVDs, increased in relation to decrease in FMD. Additionally, FMD inversely correlated with Framingham Risk score, which is a risk calculator and an index of cumulative cardiovascular risk commonly used for assessing the probability of heart attack or death from heart disease within 10 years. These results are consistent with results of previous studies demonstrating a correlation between cumulative risk factors and impaired FMD. ${ }^{4}$ These findings suggest that FMD can be used as a marker of atherosclerosis, indicating the
Figure 1 Bar graphs show flow-mediated vasodilation in subjects classified into four groups based on brachial artery diameter in each decade of age.

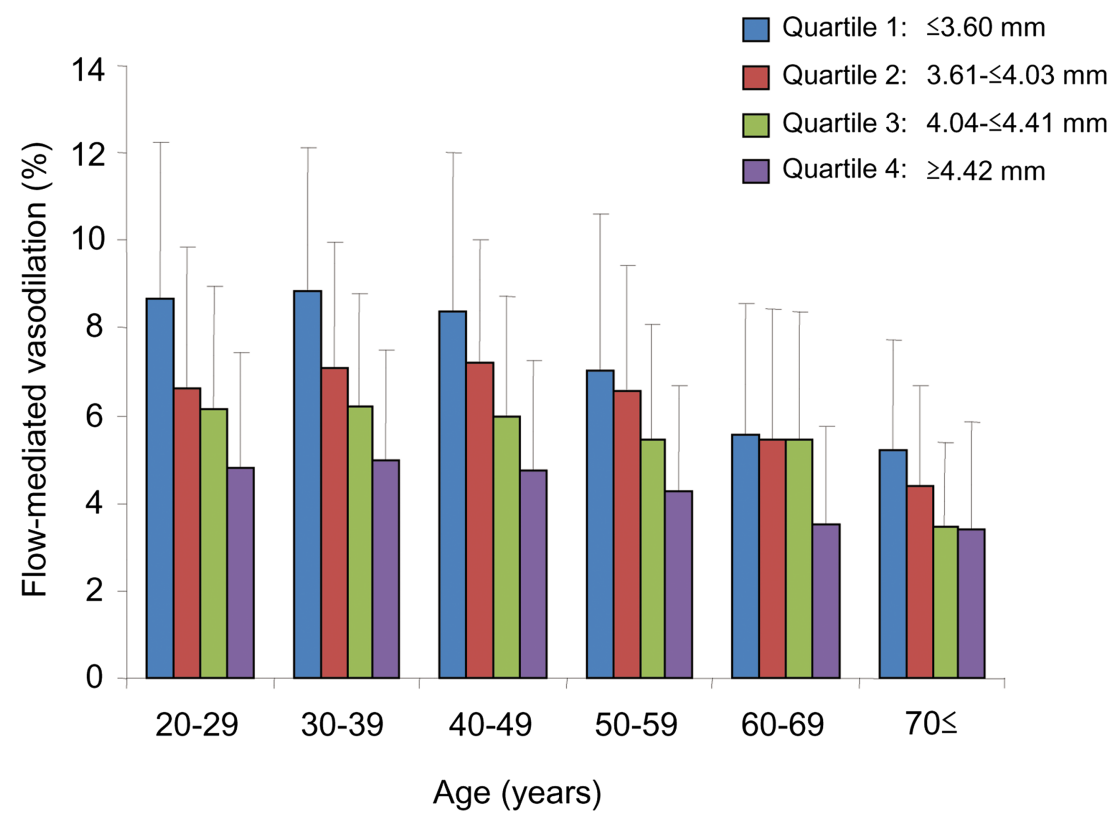

Age (years) 
possibility to provide additional information about the extent of atherosclerosis and for risk stratification in a general population.

In the present study, multivariate analysis revealed that age, male gender, systolic blood pressure and baseline brachial artery diameter were independent determinants of FMD. A correlation between aging and impaired endothelial function has been consistently shown in previous studies. ${ }^{51321}$ Although the precise mechanism by which aging is associated with endothelial dysfunction has not been fully elucidated, an imbalance of endothelium-derived vasodilators, especially NO, and reactive oxygen species, so-called oxidative stress, may be involved in age-related endothelial dysfunction. We previously demonstrated that a deficiency of tetrahydrobiopterin, an essential cofactor for NO synthase, is involved in the pathogenesis of disturbances in endothelium-dependent vasodilation related to aging through decrease in $\mathrm{NO}$ production and increase in oxidative stress. $^{22}$ In the present study, FMD in women was significantly higher than that in men until the 40s, after which it declined with no significant difference between genders from the $50 \mathrm{~s}$ in the no-risk group. The age-related decline pattern of FMD in women without cardiovascular risk factors may be, at least in part, due to female hormones, including oestrogens. The decade of the 50s at which gender-related difference in FMD disappeared in the present study was around the time of menopause. On the other hand, in the at-risk and CVD groups, there was no significant difference in FMD between men and women from the 30s, suggesting that cardiovascular risk factors diminished the protective effect of female hormones against endothelial dysfunction.

Systolic blood pressure and diastolic blood pressure were also independent determinants of FMD. It has been reported that endothelial function becomes progressively impaired as blood pressure increases and that the degree of dysfunction is related to the severity of hypertension. ${ }^{23}{ }^{24}$ Although several mechanisms, including decreased NO bioavailability, ${ }^{25}$ excessive oxidative stress induced by angiotensin $\mathrm{II},{ }^{26}$ and inflammation, ${ }^{27}$ have been proposed, the pathogenesis of the relation between hypertension and endothelial dysfunction is not fully understood. Impairment of endothelial function assessed by FMD is already present at even prehypertensive blood pressure levels. ${ }^{5}$ Additionally, it has been reported that prehypertensive subjects categorised into high-normal blood pressure (systolic pressure of 130-139 $\mathrm{mm} \mathrm{Hg}$ or diastolic pressure of $85-89 \mathrm{~mm} \mathrm{Hg}$ ) have a higher incidence of CVD than do those with optimal blood pressure (systolic pressure $<120 \mathrm{~mm} \mathrm{Hg}$ and diastolic pressure $<80 \mathrm{~mm} \mathrm{Hg}$ ). ${ }^{28}$ These findings suggest that impaired endothelial function contributed to increased cardiovascular events in subjects with high-normal blood pressure. Further studies are needed to determine the relationship between FMD and occurrence of cardiovascular events in prehypertensive subjects.

Baseline brachial artery diameter has been reported to be a potential factor that might confound interpretation of FMD. ${ }^{4} 5111216$ When the baseline diameter changes, the value of FMD expressed as the resulting percent change in diameter might be affected. FMD has been demonstrated to be proportional to hyperaemic systolic shear stress that occurs during postischaemic hyperaemia. ${ }^{29}$ Therefore, a possible explanation for the inverse correlation between FMD and baseline brachial artery diameter is that shear stress stimulus during reactive hyperaemia is greater in small arteries because of the dependence of postischaemic systolic flow on radius squared, leading to greater FMD in small arteries. ${ }^{30}$ Although previous studies have shown that FMD correlates negatively with baseline brachial artery diameter, it is unclear whether this inverse correlation is present in all decades of age. In the present study, we demonstrated that FMD decreased in relation to increase in the brachial artery diameter, and strongly correlated with the brachial artery diameter in every decade from the 20 s through the over 70 s. These findings suggest that brachial artery diameter should be taken into consideration as a confounding factor of FMD in all ages of subjects. It needs to be clarified whether brachial artery diameter per se is a mere confounding factor or a manifestation of atherosclerosis for interpretation of the value of FMD.

There were some limitations in this study. First, among the 3819 non-current smokers, we had no information for 1616 subjects $(42.3 \%)$ on whether they were ex-smokers or not. Therefore, the influence of former smoking has not been taken into account in the analysis in the present study. Second, the relationships between FMD and cardiovascular risk factors and the strong correlation between FMD and brachial artery diameter, as a confounding factor in the interpretation of the results of FMD, have been well established in previous studies. ${ }^{4} 5$ However, this study reconfirmed these associations in a large number of well-characterised subjects using automated computerised ultrasonography with an edgetracking system, which enables identification of the true peak value of FMD. The size of the study and the accuracy of the measurements validated the usefulness of this approach and provided evidence for the possibility of FMD being used as a marker of atherosclerosis.

In conclusion, FMD measured by using an ultrasound system with an edge detection and wall-tracking system is associated with cardiovascular risk factors in a general population. This study endorses the application of this method for measurement of FMD in clinical practice. Baseline brachial artery diameter correlates strongly with FMD in all decades of age. When applying the results of FMD in a clinical setting, we should take these parameters into account as potential factors that might confound interpretation of FMD. Further studies are needed to establish diagnostic criteria in order to diagnose endothelial dysfunction for detection of the early stage of vascular failure and of future cardiovascular outcomes.

\section{Key messages}

\section{What is already known on this subject?}

The relationships between flow-mediated vasodilation (FMD) and cardiovascular risk factors, and the strong correlation between FMD and brachial artery diameter, as a confounding factor in the interpretation of the results of FMD, have been well established in previous studies.

\section{What this study adds?}

In the present study, we adopted a continuous diameter assessment methodology using automated computerised ultrasonography with an edge-tracking system. In this approach, the transducer was held at the same position, and edges of the brachial artery were automatically detected and continuously tracked at the same point throughout the study. Therefore, compared with the traditional approach, this method may provide true values of peak FMD and enable more accurate and simpler measurement of FMD.

Baseline brachial artery diameter correlates strongly with FMD in all decades of age. When applying the results of FMD in a clinical setting, we should take these parameters into account as potential factors that might confound interpretation of FMD. 


\section{Author affiliations}

'Department of Cardiovascular Medicine, Hiroshima University Graduate School of Biomedical Sciences, Hiroshima, Japan

${ }^{2}$ Department of Medicine and Molecular Science, Hiroshima University Graduate School of Biomedical Sciences, Hiroshima, Japan

${ }^{3}$ Department of Cardiovascular Regeneration and Medicine, Research Institute for Radiation Biology and Medicine, Hiroshima University, Hiroshima, Japan

${ }^{4}$ Division of Regeneration and Medicine, Hiroshima University Hospital, Hiroshima, Japan

${ }^{5}$ Hirohsima International University, Hiroshima, Japan

${ }^{6}$ The Second Department of Internal Medicine, Tokyo Medical University, Tokyo, Japan

${ }^{7}$ Division of Biomedical Engineering, National Defense Medical College Research Institute, Tokorozawa, Japan

Acknowledgements We thank Megumi Wakisaka, Miki Kumijl, Kiichiro Kawano and Satoko Michiyama for their excellent secretarial assistance.

Contributors $\mathrm{TM}$ and $\mathrm{YH}$, drafting the article and conception of this study; JS, NF, $\mathrm{NI}, \mathrm{SM}, \mathrm{YI}, \mathrm{MK}, \mathrm{TM}, \mathrm{TH}, \mathrm{KN}, \mathrm{AN}$ and $\mathrm{CG}$, performing the ultrasonography; $\mathrm{KC}, \mathrm{YK}$, $\mathrm{HT}, \mathrm{BT}$ and $\mathrm{AY}$, revising the article critically for important intellectual content.

Funding This study was supported in part by a Grant-in-Aid for Scientific Research from the Ministry of Education, Science and Culture of Japan (1859081500 and 21590898) and a Grant-in-Aid of Japanese Arteriosclerosis Prevention Fund.

\section{Competing interests None.}

Patient consent Obtained.

Ethics approval Ethical Committees of each hospital.

Provenance and peer review Not commissioned; externally peer reviewed.

Open Access This is an Open Access article distributed in accordance with the Creative Commons Attribution Non Commercial (CC BY-NC 3.0) license, which permits others to distribute, remix, adapt, build upon this work non-commercially, and license their derivative works on different terms, provided the original work is properly cited and the use is non-commercial. See: http://creativecommons.org/ licenses/by-nc/3.0/

\section{REFERENCE}

1 Ross R. Atherosclerosis - an inflammatory disease. N Engl J Med 1999;340:115-26.

2 Lerman A, Zeiher AM. Endothelial function: cardiac events. Circulation 2005; 111:363-8.

3 Celermajer DS, Sorensen KE, Gooch VM, et al. Non-invasive detection of endothelial dysfunction in children and adults at risk of atherosclerosis. Lancet 1992;340:1111-15.

4 Celermajer DS, Sorensen KE, Bull C, et al. Endothelium-dependent dilation in the systemic arteries of asymptomatic subjects relates to coronary risk factors and their interaction. J Am Coll Cardiol 1994;24:1468-74.

5 Benjamin EJ, Larson MG, Keyes MJ, et al. Clinical correlates and heritability of flow-mediated dilation in the community: the Framingham Heart Study. Circulation 2004;109:613-19.

6 Idei N, Nishioka K, Soga J, et al. Vascular function and circulating progenitor cells in thromboangitis obliterans (Buerger's disease) and atherosclerosis obliterans. Hypertension 2011:57:70-8.

7 Soga J, Noma K, Hata T, et al. Rho-associated kinase activity, endothelial function, and cardiovascular risk factors. Arterioscler Thromb Vasc Biol 2011;31:2353-9.

8 Modena MG, Bonetti L, Coppi F, et al. Prognostic role of reversible endothelial dysfunction in hypertensive postmenopausal women. J Am Coll Cardiol 2002:40:505-10.
9 Gokce N, Keaney JF Jr., Hunter LM, et al. Risk stratification for postoperative cardiovascular events via noninvasive assessment of endothelial function: a prospective study. Circulation 2002;105:1567-72.

10 Yeboah J, Folsom AR, Burke GL, et al. Predictive value of brachial flow-mediated dilation for incident cardiovascular events in a population-based study: the multi-ethnic study of atherosclerosis. Circulation 2009;120:502-9.

11 Corretti MC, Anderson TJ, Benjamin EJ, et al. Guidelines for the ultrasound assessment of endothelial-dependent flow-mediated vasodilation of the brachial artery: a report of the International Brachial Artery Reactivity Task Force. J Am Coll Cardiol 2002:39:257-65.

12 Inoue $\mathrm{T}$, Matsuoka $\mathrm{H}$, Higashi $\mathrm{Y}$, et al. Flow-mediated vasodilation as a diagnostic modality for vascular failure. Hypertens Res 2008;31:2105-13.

13 Celermajer DS, Sorensen KE, Spiegelhalter DJ, et al. Aging is associated with endothelial dysfunction in healthy men years before the age-related decline in women. J Am Coll Cardiol 1994;24:471-6.

14 Wisloff U, Stoylen A, Loennechen JP, et al. Superior cardiovascular effect of aerobic interval training versus moderate continuous training in heart failure patients: a randomized study. Circulation 2007:115:3086-94.

15 Black MA, Cable NT, Thijssen DH, et al. Importance of measuring the time course of flow-mediated dilatation in humans. Hypertension 2008;51:203-10.

16 Schroeder S, Enderle MD, Baumbach A, et al. Influence of vessel size, age and body mass index on the flow-mediated dilatation (FMD\%) of the brachial artery. Int $J$ Cardiol 2000;76:219-25.

17 Expert committee on the diagnosis and classification of diabetes mellitus, American Diabetes Association: clinical practice recommendations 1999. Diabetes Care 1999;22(Suppl 1):S1-114.

18 Expert panel on detection, evaluation and treatment of high blood cholesterol in adults. Executive Summary of The Third Report of The National Cholesterol Education Program (NCEP) expert panel on detection, evaluation, and treatment of high blood cholesterol in adults (adult treatment panel III). JAMA 2001;285:2486-97.

19 Wilson PW, Castelli WP, Kannel WB. Coronary risk prediction in adults (the Framingham Heart Study). Am J Cardiol 1987;59:91G-4G.

20 Chobanian AV, Barkis GL, Black HR et al. The seventh report of the Joint National Committee on prevention, detection, evaluation, and treatment of high blood pressure. Arch Intern Med 2003;289:2560-72.

21 Herrington DM, Fan L, Drum $M$, et al. Brachial flow-mediated vasodilator responses in population-based research: methods, reproducibility and effects of age, gender and baseline diameter. J Cardiovasc Risk 2001:8:319-28.

22 Higashi Y, Sasaki S, Nakagawa K, et al. Tetrahydrobiopterin improves aging-related impairment of endothelium-dependent vasodilation through increase in nitric oxide production. Atherosclerosis 2006;186:390-5.

23 Dohi Y, Thiel MA, Buhler FR, et al. Activation of endothelial L-arginine pathway in resistance arteries. Effect of age and hypertension. Hypertension 1990;16:170-9.

24 Panza JA, Casino PR, Kilcoyne CM, et al. Role of endothelium-derived nitric oxide in the abnormal endothelium-dependent vascular relaxation of patients with essential hypertension. Circulation 1993;87:1468-74.

25 Huang $\mathrm{PL}$, Huang $\mathrm{Z}$, Mashimo $\mathrm{H}$, et al. Hypertension in mice lacking the gene for endothelial nitric oxide synthase. Nature 1995:377:239-42.

26 Higashi Y, Sasaki S, Nakagawa K, et al. Endothelial function and oxidative stress in renovascular hypertension. N Engl J Med 2002;346:1954-62.

27 Brasier AR, Recinos A III, Eledrisi MS. Vascular inflammation and the renin-angiotensin system. Arterioscler Thromb Vasc Biol 2002;22:1257-66.

28 Vasan RS, Larson MG, Leip EP, et al. Impact of high-normal blood pressure on the risk of cardiovascular disease. N Engl J Med 2001;345:1291-7.

29 Silber HA, Bluemke DA, Ouyang $P$, et al. The relationship between vascular wall shea stress and flow-mediated dilation: endothelial function assessed by phase-contrast magnetic resonance angiography. J Am Coll Cardiol 2001;38:1859-65.

30 Silber HA, Ouyang P, Bluemke DA, et al. Why is flow-mediated dilation dependent on arterial size? Assessment of the shear stimulus using phase-contrast magnetic resonance imaging. Am J Physiol Heart Circ Physiol 2005;288:H822-8. 